\title{
Elemente der Selbstanalyse in den «Studien über Hysterie»
}

Erläuterungen zum Ursprung der psychoanalytischen Technik

Von Heinz G. Schott

\section{Einleitung}

Mit meinem Thema möchte ich eine Problematik ansprechen, die die medizinische Geschichtsschreibung und die psychoanalytische Theoriebildung gleichermaßen betrifft, deren gründliche Bearbeitung jedoch bis heute aussteht. Meine Arbeit soll dazu beitragen, die auch heute noch rätselhafte Selbstanalyse Freuds ein Stück weit aufzuklären. Zunächst wäre dreierlei festzuhalten:

1. Unter «Selbstanalyse» versteht man im allgemeinen die Selbstanalyse Freuds, die der «Traumdeutung» zugrunde liegt und in diesem Buch stückweise dargestellt wird. Sie wird von verschiedenen Autoren unterschiedlich aufgefaßt: (a) als aufschlußreiches Material für die FreudBiographik (Bernfeld, 1946; Bernfeld und Cassirer-Bernfeld, 1944; Jones, 1960; Schur, 1973); (b) als Sonderform eines psychoanalytischen Prozesses, der sich zwischen Freud und Fliess abgespielt hat (Mannoni, 1967; Kohut, 1974); und (c) schließlich als Arbeitsprozeß, der sich in der «Traumdeutung» vergegenständlicht (Anzieu, 1959) ${ }^{1}$.

2. Die «Studien über Hysterie», von Breuer und Freud gemeinsam herausgegeben (1895), gehören zu den meistbeachteten Texten der Psychoanalyse. Sie werden gemäß dem Freudschen Motto von der «Entwicklung der Katharsis zur Psychoanalyse» (Freud, 1908) - als wichtigste Vorstufe zur Psychoanalyse bzw. als deren Ausgangspunkt angesehen (Brenner, 1955). Wegen des in ihnen angesprochenen Mechanismus der «Konversion» (Konversionshysterie) gehören sie bis heute zu den grundlegenden Texten der psychosomatischen Medizin (Hahn, 1977) ${ }^{2}$.

3. Es besteht Einigkeit darüber, was als Freudsche «psychoanalytische Technik» anzusehen sei, wobei man häufig auch von «klassischer Technik» spricht. Nach Greenson (1967) liegen die Hauptzüge der psychoanalytischen Technik mit den technischen Schriften Freuds zwischen den 
Jahren 1912 und 1915 fest und dienen auch heute noch als «Basis der psychoanalytischen Praxis» (S. 17) (vgl. auch Lipton, 1977). Neuerdings gibt es eine Diskussion über die Theorie der Technik, die sich von einer immer abstrakter werdenden Methapsychologie distanziert und den psychoanalytischen Prozeß selber in den Mittelpunkt der Betrachtung rückt (Cremerius, 1979; Morgenthaler, 1978).

Meine Arbeit erwächst aus einer gewissen Kritik an der oben skizzierten Behandlung der angesprochenen Gesichtspunkte: ich meine, daß die Problematik der Selbstanalyse Freuds noch keineswegs in die psychoanalytische Theoriebildung eingeholt ist, daß die «Studien über Hysterie» von ihrer Textgrundlage her noch nicht gründlich genug durchgearbeitet wurden und daß die psychoanalytische Technik noch zu wenig auf ihren Entstehungsprozeß hin befragt wurde. Offenbar machen die diesbezüglichen Diskussionen frühzeitig halt: sie orientieren sich anscheinend an Axiomen ${ }^{3}$, die zwar historisch beleuchtet werden können, aber nicht notwendigerweise historisch analysiert, aufgelöst werden müssen.

Ich möchte im folgenden die «Studien über Hysterie» so auslegen, daß wir einige Elemente der Selbstanalyse Freuds klarer bestimmen können. Dabei gehe ich von der Arbeitshypothese aus, daß wir ohne eine gründliche Reflektion der Selbstanalyse auch in unserem Verständnis der «klassischen» Technik der Psychoanalyse nicht weiterkommen. Die Selbstanalyse erscheint als wesentliches Fundament, auf der die gesamte Psychoanalyse aufbaut. Ich hoffe, einige Bausteine dieses Fundaments beschreiben zu können und damit - wenn auch nur indirekt - einen Beitrag zur aktuellen Diskussion über die Theorie der Technik zu leisten.

Die Untersuchung des Textes soll im Vordergrund stehen. Aus seiner Reichhaltigkeit habe ich einige Auszüge entnommen und einzelne Ausdrücke hervorgehoben, die für unser Thema besonders wichtig sind. Ich werde mich im folgenden auf drei Textabschnitte der «Studien über Hysterie» konzentrieren: auf die gemeinsame «Vorläufige Mitteilung» von Breuer und Freud, auf die Breuersche Krankengeschichte der Anna O. und auf Freuds Beitrag «zur Psychotherapie der Hysterie». Auf die vieldiskutierten Unterschiede zwischen Breuer und Freud sowohl in theoretischer wie in praktischer Hinsicht möchte ich hier nicht näher eingehen, sie sind schon häufig dargestellt worden (Laplanche/Pontalis, 1967, S. 131 ff.; Hirschmüller, 1979). Mir kommt es hier auf den gemeinsamen Ansatz beider Autoren an. 
Die Textinterpretation befaßt sich mit drei Problemkreisen, in denen die Elemente der Freudschen Selbstanalyse zum Vorschein kommen - einige Jahre vor ihrer praktischen Durchführung ${ }^{4}$ :

1. die Charakterisierung des Krankheitsbildes der Hysterie als Spaltung des Bewußtseins;

2. die therapeutische Technik, die sich eine «assoziative Korrektur» zum Ziel setzt; und

3. Freuds theoretisches Modell der psychotherapeutischen Arbeit, die man als Theorie der Technik bezeichnen kann.

\section{II. «Normal» und «alieniert»: die Dissoziation des Bewußtseins}

Anna O., die hysterische Patientin Breuers, leidet an einer Krankheit, die in mehrfacher Hinsicht Befremden auslöst: ihre Symptomatik befremdet die Personen in der familiären Umgebung, die behandelnden Ärzte, insbesondere Breuer, und - last but not least - die Patientin selber. In der Krankengeschichte Breuers werden die einzelnen Symptome zu Beginn der Behandlung von Breuer eindringlich aufgeführt:

«Linksseitiger Hinterkopfschmerz; Strabismus convergens (Diplopie) durch Aufregung bedeutend gesteigert; Klage über Herüberstürzen der Wand (Obliquusaffektion). Schwer analysierbare Sehstörungen; Parese der vorderen Halsmuskeln, so daß der Kopf schließlich nur dadurch bewegt wurde, daß Patientin ihn nach rückwärts zwischen die gehobenen Schultern preßte und sich mit dem ganzen Rücken bewegte. Kontraktur und Anästhesie der rechten oberen, nach einiger Zeit der rechten unteren Extremität; auch diese völlig gestreckt, adduziert und nach innen rotiert; später tritt dieselbe Affektion an der linken unteren Extremität und zuletzt am linken Arme auf, an welchem aber die Finger einigermaßen beweglich blieben. Auch die Schultergelenke beiderseits waren nicht völlig rigide. Das Maximum der Kontraktur betrifft die Muskeln des Oberarmes, wie auch später, als die Anästhesie genauer geprüft werden konnte, die Gegend des Ellbogens sich als am stärksten unempfindlich erwies. Im Beginne der Krankheit blieb die Anästhesieprüfung ungenügend, wegen des aus Angstgefühlen entspringenden Widerstandes der Patientin» (Breuer und Freud, 1895, S.21 f.).

Breuer ist nicht so sehr über diese manifesten Krankheitssymptome selber befremdet, vielmehr beeindruckt ihn die erstaunliche Beobachtung, daß «zwei ganz getrennte Bewußtseinszustände» bei der Patientin bestanden, «die sehr oft und unvermittelt abwechselten und sich im Laufe der Krankheit immer schärfer schieden» (l.c., S.22). Diese Feststellung taucht in der Krankengeschichte immer wieder in verschiedener Version auf und bezeich- 
net für Breuer offenbar das Charakteristikum der Krankheit. Er unterstreicht dies noch einmal gegen Ende der Krankengeschichte:

«Während des ganzen Krankheitsverlaufes bestanden die zwei Bewußtseinszustände nebeneinander, der primäre, in welchem Patientin psychisch ganz normal war, und der (zweite) Zustand, den wir wohl mit dem Traume vergleichen können, entsprechend seinem Reichtum an Phantasmen, Halluzinationen, den großen Lücken der Erinnerung, der Hemmungs- und Kontrollelosigkeit der Einfälle. In diesem zweiten Zustande war Patientin alieniert. Es scheint mir nun guten Einblick in das Wesen mindestens einer Art von hysterischen Psychosen zu gewähren, daß der psychische Zustand der Kranken durchaus abhängig war von dem Hereinragen dieses zweiten Zustandes in den normalen... Es ist schwer, dem Ausdrucke aus dem Wege zu gehen, die Kranke sei in zwei Persönlichkeiten zerfallen, von denen die eine psychisch normal und die andere geisteskrank war. Ich meine, daß die scharfe Trennung der beiden Zustände bei unserer Kranken ein Verhalten nur deutlich machte, das auch bei vielen anderen Hysterischen Ursache so mancher Rätsel ist. Bei Anna O.... war besonders auffallend, wie sehr die Produkte des «schlimmen Ichs», wie die Kranke selbst es nannte, ihren moralischen Habitus beeinflußten» (l.c., S. 38 f.).

Für Breuer ist dieses Nebeneinander von «normalem» und «alieniertem» Zustand ein Paradoxon; der Widerspruch zwischen Normal-Sein und Alieniert-Sein, den die Patientin durchlebt, wird von Breuer noch durch die anamnestische Feststellung verstärkt:

«Sie selbst (Anna O.-H.S.) früher stets gesund, ohne irgendein Nervosum während der Entwicklungsperiode; von bedeutender Intelligenz, erstaunlich scharfsinniger Kombination und scharfsichtiger Intuition; ein kräftiger Intellekt ...» (l.c., S. 20).

Rätselhaft ist also zunächst, wie es zu dem genannten Widerspruch der «getrennten Bewußtseinszustände» kommt. Doch dieser Widerspruch wird nicht nur von Breuer mit Befremden aufgenommen: er korreliert sie mit der Selbstbeobachtung der Patientin, die sie ihrem Arzt gegenüber in anschaulicher Art und Weise beschreiben kann. Sie nennt ihre ausgedehnten Wachträumereien «Privattheater» (l.c.).

«In ganz klaren Momenten beklagte sie die tiefe Finsternis ihres Kopfes, wie sie nicht denken könne, blind und taub werde, zwei Ichs habe, ihr wirkliches und ein schlechtes, das sie zu Schlimmem zwinge, usw.» (l.c., S.23).

«Aber so scharf die beiden Zustände getrennt waren, es ragte nicht bloß der szweite Zustands in den ersten hinein, sondern es saß, wie Patientin sich ausdrückte, mindestens häufig auch bei ganz schlimmen Zuständen in irgendeinem Winkel ihres Gehirnes ein scharfer und ruhiger Beobachter, der sich das tolle Zeug ansah» (1. c., S. 39).

Breuers Schilderung zweier getrennter Bewußtseinszustände stimmt mit der Selbstschilderung seiner Patientin überein, wobei es naheliegt anzuneh- 
men, daß gerade die Aussagen der Patientin über ihren gespaltenen Bewußtseinszustand ihn dazu gebracht haben, diesen auch objektiv festzustellen. In diesem Sinn meint Thomä, daß Anna O. «die erste Ichpsychologin war, als sie ihr schlimmes Ich entdeckte» (Thomä, 1979, S. 34).

Breuers Befunderhebung schlägt sich an zentraler Stelle in der gemeinsam mit Freud publizierten «Vorläufigen Mitteilung» von 1893 nieder. Breuer spricht in seiner Krankengeschichte von «Dissociation der geistigen Persönlichkeit» (Breuer und Freud, 1895, S.36). In der «Vorläufigen Mitteilung» ist die «Dissoziation» ein zentraler Begriff, der die «Spaltung des Bewußtseins» bezeichnet (Breuer und Freud, 1893, S.91). Der von Breuer immer wieder herausgestellte verblüffende Widerspruch zwischen «normalem» und «alieniertem» Zustand wird auch in der «Vorläufigen Mitteilung» hervorgehoben und sogar durch einen Vergleich mit der «Traumpsychose» verallgemeinert. In seinen hypnoiden Zuständen sei der Hysteriker «alieniert, wie wir es alle im Traume sind». Es lohnt sich, die Textpassage ausführlich zu lesen:

Die Erfahrungen mit den hysterischen Patientinnen beleuchten für Freud und Breuer «den Widerspruch zwischen dem Satze: ‘Hysterie ist eine Psychose`, und der Tatsache, daß man unter den Hysterischen die geistig klarsten, willensstärksten, charaktervollsten und kritischsten Menschen finden kann. In diesen Fällen ist solche Charakteristik richtig für das wache Denken des Menschen; in seinen hypnoiden Zuständen ist er alieniert, wie wir es alle im Traume sind. Aber während unsere Traumpsychosen unseren Wachzustand nicht beeinflussen, ragen die Produkte der hypnoiden Zustände als hysterische Phänomene ins wache Leben hinein.»

Der befremdende Widerspruch betrifft also nicht nur den Hysterischen, nein: «wir alle» sind von ihm betroffen! Somit kommt es zu einer eigenartigen Verquickung zwischen Normal-Sein und Alieniert-Sein: beim hysterischen Menschen ist das Fortbestehen des normalen Wachzustands ein Wesensmerkmal seiner Symptomatik, beim normalen Menschen dagegen gehört der alienierte Zustand (beim Träumen) durchaus zu seiner Normalität.

Vielleicht kann man sagen: der Hysteriker ist auch normal, der Normale ist auch alieniert.

Was aber heißt es, «alieniert» zu sein? Was bedeutet der Ausdruck «geistige Alienation» (Breuer und Freud, 1895a, S. 152)?

Sicherlich schwingt hier noch die alte Bezeichnung der alienatio mentalis für Irresein, Psychose, mit, der Pinel (1809) sein grundlegendes Werk gewidmet hat. Zunächst könnte man Alieniert-Sein mit Anders-Sein über- 
setzen, und Alienation mit "Veranderung» ${ }^{5}$ in bezug auf das normale Wachleben. Der andere Zustand wird als schlafähnlich («hypnoid»), traumähnlich («Wachträumen»), geistesabwesend («Absence») und abgespalten vom gesellschaftlichen Verkehr («Privattheater») gekennzeichnet.

Zugleich aber trägt der Ausdruck Alieniert-Sein auch die Bedeutung von Entfremdet-Sein, denn die Symptomatik der Krankheit befremdet nicht nur, sie ist Ausdruck einer Entfremdung. So beschreibt Breuer die Pathogenese als einen Prozeß der Entfremdung, der im Seelenleben der Anna O, abläuft, «wie unmittelbar diese gewohnheitsmäßige Träumerei in Krankheit überging» (Breuer und Freud, 1895, S.20), und beobachtet, wie «die Existenz eines zweiten Bewußtseinszustandes, der sich, zuerst als vorübergehende Absenz auftretend, später zur double conscience organisiert» (l.c., S. 36). Es ist interessant, daß Freud vom pathogenen Material der Hysterie aussagt, «es benehme sich wie ein Fremdkörper» (Breuer und Freud, 1895a, S.294) und diesen Vergleich sofort wieder korrigiert: «Die pathogene Organisation verhält sich nicht eigentlich wie ein Fremdkörper, sondern weit eher wie ein Infiltrat. Als das Infiltrierende muß in diesem Gleichnisse der Widerstand genommen werden» (l.c., S.295). Die Krankheit ist also nur scheinbar ein «Fremdkörper», tatsächlich aber wird er vom Organismus selber produziert. In Freuds Krankengeschichte «Fräulein Elisabeth v. R. .... verweist Freud wiederum auf den (falschen) Anschein, als sei das Leiden von einem «Fremdkörper» hervorgerufen:

«Bei Fräulein Elisabeth war mir von Anfang an wahrscheinlich, daß sie sich der Gründe ihres Leides bewußt sei, daß sie also nur ein Geheimnis, keinen Fremdkörper im Bewußtsein habe. Man mußte, wenn man sie ansah, an die Worte des Dichters denken: 〈Das Mäskchen da weissagt verborgnen Sinn»» (l.c., S. 200).

Die Alienation erzeugt den «Schein einer zweiten Persönlichkeit» (l.c., S.291). Sie läßt sich aber nicht nur als Entfremdung ${ }^{6}$, sondern auch als Selbstentfremdung fassen. Denn das Leiden wird nicht allein durch eine Schädigung von außen, ein "psychisches Trauma», gesetzt, das nun als «Fremdkörper» das Seelenleben irritiert; die Hysterie ist nicht nur ein passives Erleiden. Vielmehr wird sie durch aktive Seelenvorgänge geschaffen, wie sie Breuer bei Anna O. beobachten kann: durch Wachträume, «Privattheater», phantastische Gedankenspiele, ja: «Autohypnose» (Breuer und Freud, 1895, S. 37). Die Spaltung des Bewußtseins wird von der Patientin selber produziert, gleichsam in einem Akt der Selbstentfremdung: sie wird «alieniert» und bleibt zugleich «normal». 
Es ist nun höchst bedeutsam für uns zu sehen, daß Breuer und Freud sich mit ihren Patientinnen insofern identifizieren können, als sie an sich selber die Selbstentfremdung entdecken: nämlich in ihrer Analogisierung von Traum und Hysterie. Diese Entdeckung der eigenen Selbstentfremdung kann als erstes Element der Selbstanalyse angesehen werden.

III. "Abreagieren» und «assoziative Korrektur»: die psychische Arbeit des Therapeuten, «den Widerstand zum Schmelzen zu bringen»

Wenden wir uns nun der Technik im Kontext der «Studien über Hysterie» zu. Wir werden sehen, daß sie ein weiteres Element der Selbstanalyse enthält. Zunächst erscheinen sowohl die kathartische Methode Breuers als auch die "psychische Analyse» Freuds (Breuer und Freud, 1895a, S.273) ganz auf den Krankheitszustand der Patientinnen beschränkt zu bleiben. Der Arzt setzt bestimmte Hilfsmittel ein, um auf diese einzuwirken. Freud vergleicht dabei die Psychotherapie mit chirurgischen Operationen, beispielsweise der «Eröffnung einer eitergefüllten Höhle» (l.c., S.311). Freud gibt hier allerdings zu bedenken:

«Eine solche Analogie findet ihre Berechtigung nicht so sehr in der Entfernung des Krankhaften als in der Herstellung besserer Heilungsbedingungen für den Ablauf des Prozesses» (l.c.).

Ich habe oben die Spaltung des Bewußtseins bei der Hysterie als Selbstentfremdung charakterisiert, die vom Betroffenen selber produziert wird. Psychoanalyse besteht nicht darin, einen pathogenen «Fremdkörper» zu entfernen, sondern - in Analogie zur chirurgischen Operation - eher darin, «der Zirkulation den Weg in ein bisher abgesperrtes Gebiet zu bahnen» (l.c., S.295). Wenn Hysterie als Selbstentfremdung verstanden wird, so kann Therapie nur darin bestehen, Heilungsbedingungen zu schaffen, die die Selbstentfremdung aufheben. Dieser therapeutische Ansatz impliziert die Selbsthilfe, gewissermaßen Selbsttherapie des Patienten.

Anna O. ist nicht nur Entdeckerin ihres «schlimmen Ich» (Thomä, 1979, S. 34), sondern zugleich Erfinderin der «talking cure», des «chimney sweeping»; sie diagnostiziert nicht nur ihre Krankheit, sie therapiert sie auch. Ich meine, man ist berechtigt, hier von einer Selbstanalyse der Patientin zu sprechen, die Breuer in seiner Krankengeschichte zur Darstellung bringt. (Die objektiven Daten der neurologischen Symptomatik haben dabei nur 
sekundäre Bedeutung, was sich vor allem im Konversionsmodell niederschlägt: primär ist der psychische Konflikt, der sich im Körperlichen entlädt.)

Es ist imponierend, wie die Patientinnen im Verlauf der Psychotherapie ihren Ärzten immer vertrauter werden. Wir sagten oben, daß das hysterische Alieniert-Sein für Breuer und Freud theoretisch nachvollziehbar war: sie verweisen auf die «Traumpsychose», die jeder an sich selber erlebt. Darüber hinaus aber ist auch der therapeutische Vorgang im Patienten, etwa die «talking cure» der Anna O., für die Therapeuten nachvollziehbar. Die Vorstellung, daß die Krankheit durch einen eingeklemmten Affekt bedingt ist und durch ein «Abreagieren» desselben therapiert werden kann, ist dem alltäglichen Leben vertraut. Die heilende Wirkung dieser «Methode der Psychotherapie» erscheint den Autoren durchaus verständlich:

«Sie [die Methode] hebt die Wirksamkeit der ursprünglich nicht abreagierten Vorstellung dadurch auf, daß sie dem eingeklemmten Affekte derselben den Ablauf durch die Rede gestattet, und bringt sie zur assoziativen Korrektur, indem sie dieselbe ins normale Bewußtsein zieht ...» (Breuer und Freud, 1893, S.97, im Text gesperrt gedruckt).

Dieses therapeutische Prinzip der «assoziativen Korrektur» hat ihr Analogon in der assoziativen Leistung des normalen Menschen, wenn dieser eine Kränkung zu verkraften hat.

«Die Erinnerung an eine Kränkung wird korrigiert durch Richtigstellung der Tatsachen, durch Erwägungen der eigenen Würde u. dgl., und so gelingt es dem normalen Menschen, durch Leistungen der Assoziation den begleitenden Affekt zum Verschwinden zu bringen» (l.c., S. 88).

Nicht nur die Therapie des «Abreagierens» in Form der «assoziativen Korrektur» findet ihre Entsprechung im normalen Leben, sondern auch das Wesen der Hysterie selber. Denn im Mittelpunkt der Betrachtung der Pathogenese steht «das affizierende Ereignis» der «Kränkung». Entscheidend dafür, ob der betroffene Mensch hysterisch wird oder normal bleibt, ist der Umstand, ob er sich «austoben, ausweinen» kann oder aber die Reaktion auf die Kränkung unterdrückt und sie schweigend erduldet. So imponiert den Autoren die Hysterie als nicht verkraftete Kränkung, an deren Erinnerung der Affekt haftet: «der Hysterische leide größtenteils an Reminiszenzen» lautet die Formel (Breuer und Freud, 1893, S.86, im Text gesperrt gedruckt). Der Terminus «Reminiszenz» deutet auf den Charakter dieser virulenten Erinnerung hin: sie irritiert das Seelenleben als Rückbleibsel, als Quasi-Fremdkörper. 
Das «Abreagieren» ist das übergreifende Modell der therapeutischen Technik: der normale Mensch hilft sich sozusagen selber, es stehen ihm «vom Weinen bis zum Racheakt» verschiedene Formen der Reaktion zur Verfügung. Der Hysteriker dagegen kann sich nicht abreagieren und wird gerade durch dieses Unvermögen zum Hysteriker. Seine Therapie besteht nun darin, daß er sich nachträglich abreagieren lernt. Ich möchte nun das betreffende Zitat in voller Länge wiedergeben, aus dem hervorgeht, wie das normale Abreagieren zum Vorbild der psychotherapeutischen Technik wird (Hervorhebung im Original wird hier kursiv gesetzt).

«Das Verblassen oder Affektloswerden einer Erinnerung hängt von mehreren Faktoren ab. Vor allem ist dafür von Wichtigkeit, ob auf das affizierende Ereignis energisch reagiert wurde oder nicht. Wir verstehen hier unter Reaktion die ganze Reihe willkürlicher und unwillkürlicher Reflexe, in denen sich erfahrungsgemäß die Affekte entladen: vom Weinen bis zum Racheakt. Erfolgt diese Reaktion in genügendem Ausmaße, so schwindet dadurch ein großer Teil des Affektes; unsere Sprache bezeugt diese Tatsache der täglichen Beobachtung durch die Ausdrücke ssich austoben, ausweinens u. dgl. Wird die Reaktion unterdrückt, so bleibt der Affekt mit der Erinnerung verbunden. Eine Beleidigung, die vergolten ist, wenn auch nur durch Worte, wird anders erinnert, als eine, die hingenommen werden mußte. Die Sprache anerkennt auch diesen Unterschied in den psychischen und körperlichen Folgen und bezeichnet höchst charakteristischerweise eben das schweigend erduldete Leiden als 〈Kränkung〉. - Die Reaktion des Geschädigten auf das Trauma hat eigentlich nur dann eine völlig «kathartisches Wirkung, wenn sie eine adäquate Reaktion ist, wie die Rache. Aber in der Sprache findet der Mensch ein Surrogat für die Tat, mit dessen Hilfe der Affekt nahezu ebenso <abreagiert) werden kann. In anderen Fällen ist das Reden eben selbst der adäquate Reflex, als Klage und als Aussprache für die Pein eines Geheimnisses (Beichte!) Wenn solche Reaktion durch Tat, Worte, in leichtesten Fällen durch Weinen nicht erfolgt, so behält die Erinnerung an den Vorfall zunächst die affektive Betonung.

Das «Abreagieren` ist indes nicht die einzige Art der Erleichterung, welche dem normalen psychischen Mechanismus des Gesunden zur Verfügung steht, wenn er ein psychisches Trauma erfahren hat. Die Erinnerung daran tritt, auch wenn sie nicht abreagiert wurde, in den großen Komplex der Assoziation ein, sie rangiert dann neben anderen, vielleicht ihr widersprechenden Erlebnissen, erleidet eine Korrektur durch andere Vorstellungen. Nach einem Unfalle z. B. gesellt sich zu der Erinnerung an die Gefahr und zu der (abgeschwächten) Wiederholung des Schreckens die Erinnerung des weiteren Verlaufs, der Rettung, das Bewußtsein der jetzigen Sicherheit. Die Erinnerung an eine Kränkung wird korrigiert durch Richtigstellung der Tatsachen, durch Erwägungen der eigenen Würde u. dgl., und so gelingt es dem normalen Menschen, durch Leistungen der Assoziation den begleitenden Affekt zum Verschwinden zu bringen» (Breuer und Freud, 1893, S. 87 f.).

Der «normale Mensch» kann sich abreagieren, indem er sich gleichsam einer Technik der befreienden Assoziation bedient, die beim Hysterischen wegen des «Assoziationswiderstandes» nicht funktioniert. Die therapeutische 
Aufgabe des Arztes besteht darin, diesen Widerstand zu überwinden, eine assoziative Korrektur zu erzwingen.

Wenden wir uns nun den Freudschen Überlegungen «zur Psychotherapie der Hysterie» zu. Durch seine therapeutischen Erfahrungen mit hysterischen Patienten habe er den Eindruck gewonnen,

«es würde in der Tat möglich sein, die doch sicherlich vorhandenen pathogenen Vorstellungsreihen durch bloßes Drängen zum Vorschein zu bringen, und da dieses Drängen mich Anstrengung kostete und mir die Deutung nahelegte, ich hätte einen Widerstand zu überwinden, so setzte sich mir der Sachverhalt ohne weiteres in die Theorie um, daß ich durch meine psychische Arbeit eine psychische Kraft bei dem Patienten zu überwinden habe, die sich dem Bewußtwerden (Erinnern) der pathogenen Vorstellungen widersetze. Ein neues Verständnis schien sich mir nun zu eröffnen, als mir einfiel, dies dürfte wohl dieselbe psychische Kraft sein, die bei der Entstehung des hysterischen Symptoms mitwirkt und damals das Bewußtwerden der pathogenen Vorstellung verhindert habe» (Breuer und Freud, 1895a, S. 268).

Freud formuliert in diesem Zitat erstmals seine Theorie der Technik. Er geht von seiner eigenen therapeutischen Kraftanstrengung aus, die ihm die «Deutung nahelegte, ich hätte einen Widerstand zu überwinden», die er dann in die «Theorie» umsetzt. Diese Theorie stellt die «psychische Arbeit» des Therapeuten in den Mittelpunkt, durch die der Widerstand des Patienten überwunden werden muß. Der Therapeut hat den Kampf gegen die Macht des «Assoziationswiderstandes» aufzunehmen, die Technik wird als Kampfmittel bestimmt. Die Aufgabe des Therapeuten besteht darin, «diesen Assoziationswiderstand durch psychische Arbeit zu überwinden».

«Solche Leistung erfolgt zuerst durch «Drängen〉, Anwendung eines psychischen Zwanges, um die Aufmerksamkeit der Kranken auf die gesuchten Vorstellungsspuren zu lenken ... Man darf nicht vergessen, da $\beta$ es sich hier überall um quantitative Vergleichung, um den Kampf zwischen verschieden starken oder intensiven Motiven handelt. Dem <Assoziationswiderstande> bei einer ernsthaften Hysterie ist das Drängen des fremden und der Sache unkundigen Arztes an Macht nicht gewachsen. Man muß auf kräftigere Mittel sinnen.

Da bediene ich mich denn zunächst eines kleinen technischen Kunstgriffes. Ich teile dem Kranken mit, daß ich im nächsten Momente einen Druck auf seine Stirne ausüben werde, versichere ihm, daß er während dieses ganzen Druckes eine Erinnerung als Bild vor sich sehen oder als Einfall in Gedanken haben werde, und verpflichte ihn dazu, dieses Bild oder diesen Einfall mir mitzuteilen, was immer das sein möge» (l. c., S.269f.).

Freud bezeichnet dieses therapeutische Vorgehen als «Technik des Drängens und der Druckprozedur» (S.285), wobei er vor allem auf die Wirksamkeit der letzteren verweist. Dieser Kunstgriff sei das «Suggestivste und Bequemste», 
wodurch «ich [Freud] ... die Aufmerksamkeit des Kranken von seinem bewußten Suchen und Nachdenken, kurz von alledem, woran sich sein Willen äußern kann, dissoziiere, ähnlich, wie es sich wohl beim Starren in eine kristallene Kugel u. dgl. vollzieht» (S.271) ${ }^{7}$.

In diesem Zusammenhang erscheint es mir interessant, den späteren technischen Grundbegriff der Gegenübertragung zu berücksichtigen. Gewöhnlich wird darauf hingewiesen, daß die Bedeutung der Gegenübertragung noch unbekannt gewesen sei, weswegen der Arzt beim psychotherapeutischen Prozeß in die größten Schwierigkeiten geraten sei, wie z. B. Breuer bei der Behandlung der Anna O. (Hirschmüller 1979; Freeman, 1972). Definiert man heute ganz allgemein die Gegenübertragung als «Gesamtheit der unbewußten Reaktionen des Analytikers auf die Person des Analysanden» (Laplanche/Pontalis, 1967, S.164), so gibt es in der oben genannten «Technik des Drängens und der Druckprozedur» sicherlich nichts Vergleichbares, da das Unbewußte des Analytikers nicht mitbedacht wird. Wir müßten hier eher von einer bewußten Aktion des Arztes sprechen, den Widerstand des Patienten zu überwinden: also von einer Übertragung psychischer Kraft durch "psychische Arbeit». Wenn der Therapeut Druck ausübt und seinen Patienten drängt, überträgt er gleichsam seine Kraft auf dessen unterdrückten und vom Bewußtsein dissoziierten Assoziationsbereich, der alleine zu schwach wäre, zum Bewußtsein vorzudringen. Diese «assoziative Korrektur» könnte man vielleicht mit einer orthopädischen Maßnahme vergleichen, die mit Druckausübung eine dislozierte Fraktur korrigiert.

Insofern sich die Kraftübertragung des Arztes gegen die Macht des Widerstandes beim Patienten wendet, gewissermaßen therapeutisch auf diese reagiert, könnte man jedoch auch von einer Gegenübertragung sprechen. Diese Art der Gegenübertragung in der Technik des Drängens ist ein Kunstgriff, der sich des Hypnotismus und der Suggestion bedient. Der Arzt überbrückt die bewußte Distanz zum Patienten, kann aber keine Brücke schlagen vom eigenen Unbewußten zu dem des Patienten, genauer gesagt: diese nicht technisch verwerten.

Freud befaßt sich schon in den «Studien über Hysterie» eingehend mit der «Übertragung auf den Arzt». Sie wird freilich nur in ihrer negativen Bedeutung gesehen, als ein «Hindernis» für die Therapie. Dieses Hindernis trete u. a. dann auf,

«wenn die Kranke sich davor erschreckt, daß sie aus dem Inhalte der Analyse auftauchende peinliche Vorstellungen auf die Person des Arztes überträgt ... Die Übertragung 
auf den Arzt geschieht durch falsche Verknüpfung. Ich muß hier wohl ein Beispiel anführen: Ursprung eines gewissen hysterischen Symptoms war bei einer meiner Patientinnen der vor vielen Jahren gehegte und sofort ins Unbewußte verwiesene Wunsch, der Mann, mit dem sie damals ein Gespräch führte, möchte doch herzhaft zugreifen und ihr einen Kuß aufdrängen. Nun taucht einmal nach Beendigung einer Sitzung ein solcher Wunsch in der Kranken in bezug auf meine Person auf; sie ist entsetzt darüber, verbringt eine schlaflose Nacht und ist das nächste Mal, obwohl sie die Behandlung nicht verweigert, doch ganz unbrauchbar zur Arbeit ... Es war also so zugegangen: Es war zuerst der Inhalt des Wunsches im Bewußtsein der Kranken aufgetreten, ohne die Erinnerungen an die Nebenumstände, die diesen Wunsch in die Vergangenheit verlegen konnten; der nun vorhandene Wunsch wurde durch den im Bewußtsein herrschenden Assoziationszwang mit meiner Person verknüpft, welche ja die Kranke beschäftigen darf, und bei dieser Mesalliance - die ich falsche Verknüpfung heiße - wacht derselbe Affekt auf, der seinerzeit die Kranke zur Verweisung dieses unerlaubten Wunsches gedrängt hat» (Breuer und Freud, 1895a, S.309).

Die positive Bedeutung der Übertragung für den analytischen Prozeß wird nur angedeutet. Als ein Mittel, den Widerstand zu überwinden, erörtert Freud «ein affektives Moment, die persönliche Geltung des Arztes» (l.c., S.286).

«Das ist hier nicht anders als sonst in der Medizin, und man wird keiner therapeutischen Methode zumuten dürfen, auf die Mitwirkung dieses persönlichen Moments gänzlich zu verzichten.»

Die Übertragung als terminus technicus mit seiner spezifischen Bedeutung ist hier noch unbekannt (Laplanche/Pontalis, S. $550 \mathrm{ff}$.). Ein gewisses «Maß an Sympathie», eine «geduldige Freundlichkeit des Arztes», sind die Voraussetzungen für die Psychotherapie der Hysterie.

«Abreagieren» und «assoziative Korrektur» sind die zwei theoretischen Pfeiler der Psychotherapie der Hysterie. Der Kampf gegen den Widerstand als «Kampf um die Erinnerung» (Mitscherlich, 1975) ist psycho-energetischer Kraftakt und logische Assoziationsarbeit in einem. Die Befreiung des eingeklemmten Affekts geht einher mit der Überwindung der Dissoziation des Bewußtseins.

C.G.Jung verkennt in seiner Schrift «Der therapeutische Wert des Abreagierens» die Theorie der Technik, wie sie in den «Studien über Hysterie» dargestellt wird. Er unterstellt nämlich Breuer und Freud, daß sie über der Technik des «Abreagierens» die Behebung der Dissoziation vergäBen und beruft sich dabei auf McDougall:

«Wenn McDougall betont, daß nicht die durch Affekte verursachte Spannung, sondern die Dissoziation der Psyche der Hauptfaktor einer Neurose ist, und daß infolgedessen das 
therapeutische Problem nicht in erster Linie im Abreagieren besteht, sondern in der Behebung der Dissoziation, so hat er wohl das Wesentliche getroffen ...

Von diesem Gesichtspunkt aus erscheint das Abreagieren in einem gänzlich anderen Licht, nämlich als ein Versuch, den autonomen Komplex wieder zu integrieren, d.h. ihn dem Bewußtsein allmählich als einen Bestandteil einzuverleiben, indem die traumatische Situation einmal oder mehrere Male wieder erlebt wird» (Jung, 1921, S. 139 f.).

Jung spricht nun des weiteren davon, «daß die bloße Wiederholung der Erfahrung keine heilende Wirkung besitzt; sie muß in Gegenwart des Arztes wiederholt werden» (l.c., S.140). Damit spielt Jung die Bedeutung des «Abreagierens» für das Seelenleben des «normalen» Menschen herunter: das Abreagieren ist - wie ich oben herausgestellt habe - der Grundmechanismus, mit dem der Normale auf eine Kränkung reagiert, ja, seine Gesundheit erweist sich gerade darin, daß er sich abzureagieren vermag. «So gelingt es dem normalen Menschen, durch Leistungen der Assoziation den begleitenden Affekt zum Verschwinden zu bringen» (Breuer und Freud, 1893, S. 88).

Anders als Freud, der durch die "psychische Arbeit» des Arztes den Widerstand des Patienten überwinden will, möchte Jung dem Patienten eine «moralische Stütze gegen den sonst nicht zu bewältigenden Affekt» geben:

Der Patient stehe somit «nicht länger allein im Kampfe gegen diese elementaren Mächte, sondern ein Mensch [der Arzt], dem er Vertrauen entgegenbringt, steht ihm zur Seite und verleiht ihm dadurch moralische Kraft, deren er bedarf, um die Tyrannei der unkontrollierbaren Emotionen zu bekämpfen. Auf diese Weise wird sein Bewußtsein gestärkt, bis er den Komplex zu integrieren vermag ...» (Jung, 1921, S. 140 f.).

IV. Das Modell der «Schichtung» und der «logische Faden» der Analyse: durch die «Enge des Bewußtseins» zur «Weite des Ichs»

Nachdem wir die ursprüngliche Freudsche Technik der Psychotherapie, das «Drängen» und die «Druckprozedur» kennengelernt haben, möchte ich nun die theoretische Fassung dieser Technik näher betrachten. Wir werden sehen, daß hier nicht nur ein praktisches Verfahren theoretisch beschrieben, sondern auch umgekehrt die Theorie der Hysterie selber praktisch dargestellt wird. Es kommt sozusagen zu einer Verschränkung von Theorie der Technik und Technik der Theorie, von «Deutungskunst» (Freud, 1923, S. 215) und «Darstellungskunst» (Freud, 1900, S. 593) ${ }^{8}$.

Freud entwirft ein Modell der «Schichtung» des «pathogenen Materials» der Hysterie und stellt damit die Krankheitsanordnung in einem morpholo- 
gisch-geologischen Bild dar. Dieses Konstrukt könnte man vielleicht den «hysterischen Apparat» nennen, der dem psychotherapeutischen Verfahren den Rahmen setzt und den Weg anweist: es ist eine theoretische Hilfsvorstellung, um die Technik der Psychotherapie einsichtig zu machen. Freud versucht also, mit einer bestimmten Methode der Darstellung die Technik der Behandlung zu bestimmen. Wollen wir hier die Termini «Darstellungskunst» und «Deutungskunst» verwenden, so können wir sagen: im Modell der «Schichtung» stellt Freud künstlich dar, wie anhand des «logischen Fadens» die hysterische Symptomatik kunstgerecht gedeutet werden kann. Das ausführliche Zitat lautet:

Das psychische Material einer solchen Hysterie stellt sich nun dar als ein mehrdimensionales Gebilde von mindestens dreifacher Schichtung. Ich hoffe, ich werde diese bildliche Ausdrucksweise bald rechtfertigen können. Es ist zunächst ein Kern vorhanden von solchen Erinnerungen (an Erlebnisse oder Gedankengänge), in denen das traumatische Moment gegipfelt oder die pathogene Idee ihre reinste Ausbildung gefunden hat. Um diesen Kern herum findet man eine oft unglaublich reichliche Menge von anderem Erinnerungsmaterial, das man bei der Analyse durcharbeiten muß, in, wie erwähnt, dreifacher Anordnung. Erstens ist eine lineare chronologische Anordnung unverkennbar, die innerhalb jedes einzelnen Themas statthat. Als Beispiel für diese zitiere ich bloß die Anordnung in Breuers Analyse der Anna O. Das Thema sei das des Taubwerdens, des Nichthörens; das differenzierte sich dann nach sieben Bedingungen, und unter jeder Überschrift waren zehn bis über hundert Einzelerinnerungen in chronologischer Reihenfolge gesammelt. Es war, als ob man ein wohl in Ordnung gehaltenes Archiv ausnehmen würde. In der Analyse meiner Patientin Emmy v. N... sind ähnliche, wenn auch nicht so vollzählig dargestellte Erinnerungsfaszikel enthalten: sie bilden aber ein ganz allgemeines Vorkommnis in jeder Analyse, treten jedesmal in einer chronologischen Ordnung auf, die so unfehlbar verläßlich ist wie die Reihenfolge der Wochentage oder Monatsnamen beim geistig normalen Menschen, und erschweren die Arbeit der Analyse durch die Eigentümlichkeit, daß sie die Reihenfolge ihrer Entstehung bei der Reproduktion umkehren; das frischeste, jüngste Erlebnis des Faszikels kommt als «Deckblatt» zuerst und den Schluß macht jener Eindruck, mit dem in Wirklichkeit die Reihe anfing.

Ich habe die Gruppierung gleichartiger Erinnerungen zu einer linear geschichteten Mehrheit, wie es ein Aktenbündel, ein Paket u. dgl. darstellt, als Bildung eines Themas bezeichnet. Diese Themen nun zeigen eine zweite Art von Anordnung; sie sind, ich kann es nicht anders ausdrücken, konzentrisch um den pathogenen Kern geschichtet. Es ist nicht schwer zu sagen, was diese Schichtung ausmacht, nach welcher ab- oder zunehmenden Größe diese Anordnung erfolgt. Es sind Schichten gleichen, gegen den Kern hin wachsenden Widerstandes und damit Zonen gleicher Bewußtseinsveränderung, in denen sich die einzelnen Themen erstrecken. Die periphersten Schichten enthalten von verschiedenen Themen jene Erinnerungen (oder Faszikel), die leicht erinnert werden und immer klar bewußt waren; je tiefer man geht, desto schwieriger werden die auftauchenden Erinnerungen erkannt, bis man nahe am Kerne auf solche stößt, die der Patient noch bei der Reproduktion verleugnet. Diese Eigentümlichkeit der konzentrischen Schichtung des 
pathogenen psychischen Materials ist es, die dem Verlaufe solcher Analysen ihre, wie wir hören werden, charakteristischen Züge verleiht. Jetzt ist noch eine dritte Art von Anordnung zu erwähnen, die wesentlichste, über die am wenigsten leicht eine allgemeine Aussage zu machen ist. Es ist die Anordnung nach dem Gedankeninhalte, die Verknüpfung durch den bis zum Kerne reichenden logischen Faden, der einem in jedem Falle besonderen, unregelmäßigen und vielfach abgeknickten Weg entsprechen mag. Diese Anordnung hat einen dynamischen Charakter, im Gegensatze zum morphologischen der beiden vorerst erwähnten Schichtungen. Während letztere in einem räumlich ausgeführten Schema durch starre, bogenförmige und gerade Linien darzustellen wären, müßte man dem Gange der logischen Verkettung mit einem Stäbchen nachfahren, welches auf den verschlungenen Wegen aus oberflächlichen in tiefe Schichten und zurück, doch im allgemeinen von der Peripherie her zum zentralen Kerne vordringt und dabei alle Stationen berühren muß, also ähnlich wie das Zickzack der Lösung einer Rösselsprungaufgabe über die Felderzeichnung hinweggeht (Breuer und Freud, 1895a, S. 292 f.).

Die Schichtung des pathogenen Materials ist also dreifach angeordnet: chronologisch («Erinnerungsfaszikel»), konzentrisch (Widerstandszonen) und dynamisch («logische Verkettung»). Diese letztgenannte «dynamische» Anordnung sei die «wesentlichste». Genauer betrachtet ist sie selber gar nicht als «Schichtung» vorstellbar, vielmehr beschreibt sie einen komplizierten Lösungsweg, um durch die Schichtungen hindurch zum «pathogenen Kern» vorzudringen. Freud vergleicht diesen Weg mit dem «Zickzack einer Rösselsprungaufgabe» und meint im weiteren:

«Der logische Zusammenhang entspricht nicht nur einer zickzackförmig geknickten Linie, sondern vielmehr einer verzweigten, und ganz besonders einem konvergierenden Liniensystem ... in den Kern münden in der Regel mehrere unabhängig voneinander verlaufende oder durch Seitenwege stellenweise verbundene Fäden ein» (S.293f.).

Freud versucht hiermit, im Modell der Schichtung die Organisation der Krankheit mit einem morphologischen Schema zu erklären, er entwirft gewissermaßen einen «Apparat», an dessen Struktur er die Funktionsweise seiner psychotherapeutischen Technik erläutert. Ich möchte nun im folgenden diese schematische Beschreibung in einer eigenen Skizze darstellen ${ }^{9}$.

Ich meine, daß diese Skizze hilfreich sein kann, um die Freudsche Theorie der Technik im Kontext der Hysterie-Studien zu veranschaulichen. Die Organisation der Hysterie insgesamt gehört einem vom frei verfügbaren Bereich der Assoziation abgesperrten Gebiet an, das von diesem «dissoziiert» ist. Sie läßt sich in ein Koordinatenkreuz eintragen, dessen Schnittpunkt der pathogene Kern ist («psychisches Trauma»). Die chronologische Anordnung besteht aus einer Schichtung von Erinnerungsblättern, die quer zur Zeitachse liegen; die konzentrische Anordnung umhüllt den pathogenen 


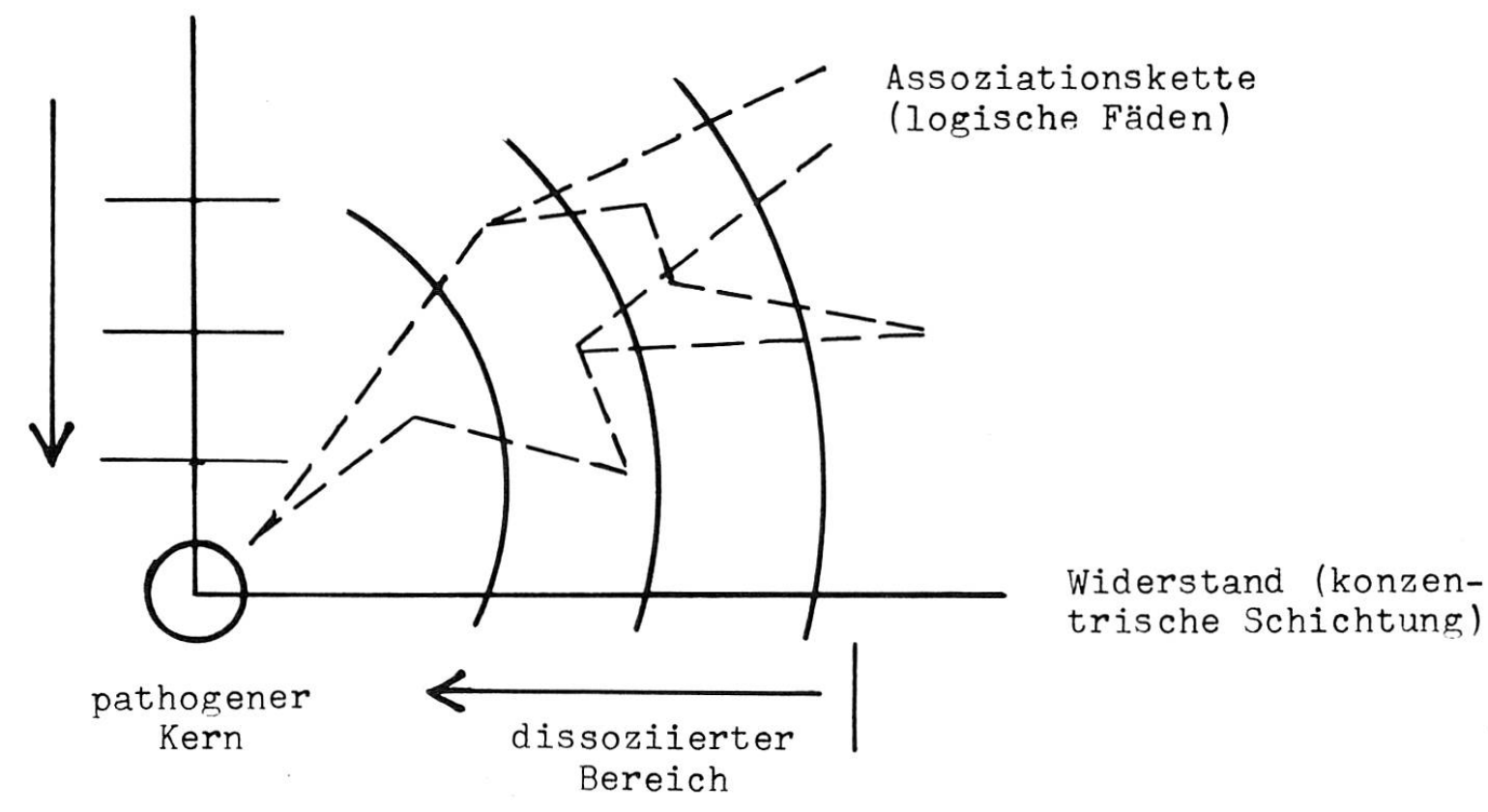

Kern, wobei der Widerstand mit zunehmender Entfernung von diesem abnimmt (Widerstandsachse). Der «logische Faden» beschreibt nun, wie im analytischen Prozeß vom Assoziationsbereich her in den dissoziierten Bereich eingedrungen wird, um diesen für die Zirkulation der Assoziation aufzuschließen. Der «logische Faden» setzt an den Erlebnissen der Gegenwart an («Deckblatt»), bzw. an Erinnerungen, die direkt wahrgenommen werden können und auf denen noch kein Widerstand lastet. Im Fortgang der Assoziationsarbeit kommt es zu einem «Liniensystem», einer «Verkettung», die sich insgesamt auf den pathogenen Kern hin fortspinnt. Die Bewußtseinsveränderung, die diesem Vorgang entspricht, wird dabei doppelt bestimmt: als Überwindung eines zunehmend stärker werdenden Widerstandes und als ein schrittweises Aufblättern der Vergangenheit.

Wie wir gehört haben, besteht nach Freud die Aufgabe des Arztes darin, «den Widerstand zum Schmelzen zu bringen». Ein Blick auf unsere Skizze macht nun einsichtig, daß die Widerstandsbarrieren den pathogenen Kern der Hysterie abschirmen, genauer gesagt: daß sie das Wesen der Hysterie ausmachen. Denn sie sperren ein Gebiet aus dem frei verfügbaren Assoziationsbereich ab, unterbinden die «Zirkulation». Die Krankheit erscheint nur als «Fremdkörper», in Wirklichkeit aber gehört sie zum eigenen Körper, und erhält nur durch ihre Ausgrenzung den Anschein der Fremdheit. 
Ziel der Technik ist es, den ausgegrenzten, d.h. dissoziierten Bereich der Assoziationen wieder an die allgemeine Zirkulationssphäre anzuschließen. Die Kunstfertigkeit der Technik besteht darin, das pathogene Material durch die «Enge des Bewußtseins» hindurchzuzwängen, so daß sich nach vollendeter Arbeit dem Beobachter die Frage stellt: «Wie kam ein solches Kamel [das pathogene Material] durch das Nadelöhr?» (l.c., S.295). Die Arbeit muß diesen «Engpaß» überwinden, denn eine «im Durchbruch befindliche Erinnerung bleibt vor dem Kranken stehen, bis er sie in die Weite des Ichs aufgenommen hat.»

«Die ganze räumlich ausgedehnte Masse des pathogenen Materials wird so durch eine enge Spalte durchgezogen, langt also, wie in Stücke oder Bänder zerlegt, im Bewußtsein an. Es ist die Aufgabe des Psychotherapeuten, daraus die vermutete Organisation wieder zusammenzusetzen. Wen noch nach Vergleichen gelüstet, der mag sich hier an ein Geduldspiel erinnern» (l.c., S.296).

Das therapeutische Motto lautet also: durch die Enge des Bewußtseins zur Weite des Ich. Die Redeweise Freuds von der «Weite des Ichs» erinnert an den Terminus der «Stärke des Ichs» (Freud, 1937, S. 69f.) und das entsprechende Motto der späteren Ich-Psychologie: «Wo Es war, soll Ich werden» (Freud, 1933, S.86). Der Analytiker zerlegt den Knäuel des pathogenen Materials, fädelt einzelne Fäden ins Nadelöhr des Bewußtseins ein, zeiht sie hindurch in die «Weite des Ichs» und verknüpft sie hinterher zum Deutungsmuster. In diesem technischen Modell spielt der Analytiker eine aktive Rolle, man könnte sogar sagen, daß Freud hier eine Art «aktive Technik» beschreibt, freilich in anderem Sinne als Ferenczi. (Grunberger charakterisiert übrigens Ferenczis «Abweichung» von Freud als eine «Rückkehr zu Freud vor der Psychoanalyse», als «eine Rückkehr zur kathartischen Technik kombiniert im übrigen mit der Technik der Suggestion»; siehe Grunberger, 1974, S. 115.)

Was Freud hier als Behandlungsmethode für die Hysterie vorführt, findet sich in der «Traumdeutung» wieder als Technik der Selbstanalyse. Es gibt für ihn weder theoretisch noch praktisch einen Hinderungsgrund, bei der Selbstanalyse dieselbe Technik anzuwenden wie bei der Psychotherapie der Hysterikerin. Die Zweipersonen-Beziehung ist nicht konstitutiv für das Modell der «Schichtung» (siehe unsere Skizze), das sich somit durchaus auch für die selbstanalytische Traumdeutungsarbeit verwenden läßt. Konstitutiv dagegen ist die «Spaltung des Bewußtseins» einer Person, die den Schein einer «zweiten Persönlichkeit» in sich trägt. Aus diesem Tatbestand leitet sich die Notwendigkeit der therapeutischen Interaktion zwischen Arzt und 
Patient ab: weil der Hysteriker es alleine nicht schafft, den Widerstand zu überwinden, muß ihm der Arzt unter die Arme greifen. Besitzt aber jemand genügend Kraft, d.h. ist jemand «normal» genug, so kann er den Widerstand durch eigene Arbeit überwinden: wie Freud, der seine eigenen Träume deutet, sich selber analysiert.

\section{Schlu $\beta$}

Ich habe versucht, einige wesentliche Elemente der Selbstanalyse am Text der «Studien über Hysterie» herauszuarbeiten. Sie bilden die Voraussetzungen für jene Durchführung der Selbstanalyse, wie sie im Briefwechsel mit Fliess dokumentiert wird und schließlich in der «Traumdeutung» zur Darstellung kommt. Diese Elemente als Voraussetzungen der später durchgeführten Selbstanalyse Freuds lassen sich in folgenden Thesen zusammenfassen:

1. Der hysterische Zustand beruht auf einer Selbstentfremdung, die jeder an sich selber beobachten kann - wenn auch nur im Traum («Traumpsychose»).

2. Die therapeutische Behandlungsmethode beruht auf Prinzipien, die auch der «normale» Mensch zur Verarbeitung von Kränkungen bei sich anwendet («Abreagieren», «assoziative Korrektur»).

3. Die Theorie der Technik entwickelt Freud am Modell der «Schichtung» des hysterischen Materials. Sie gilt für die Patientenbehandlung ebenso wie für den selbstanalytischen Prozeß, wie er in der «Traumdeutung» beschrieben wird.

Allgemein könnte man sagen, daß der Umgang mit den hysterischen Patientinnen die entscheidende Voraussetzung für Freud geschaffen hat, mit den eigenen Träumen umzugehen. Dies gilt in zweierlei Hinsicht: zum einen wird die Einsicht in die Bewußtseinsspaltung der Hysterikerin zur theoretischen Voraussetzung für das selbstanalytische Begreifen des eigenen psychischen Konflikts, zum anderen wird der therapeutische Umgang mit den Patientinnen zur praktischen Voraussetzung für die Erfahrung mit dem eigenen unterdrückten Seelenleben. Somit repräsentieren die HysterieStudien und die in ihnen entwickelte Technik ein theoretisches wie praktisches Vorverständnis der Selbstanalyse, von der die «Traumdeutung» Zeugnis ablegt. In den Hysterie-Studien kommen die Elemente der Selbst- 
analyse nicht nur theoretisch zum Vorschein: sie sind vielmehr bereits Ergebnis einer psychotherapeutischen Praxis, die von Freud bei der Selbstanalyse dann auf die eigene Person angewandt wird.

Es müßte eigens untersucht werden, wie die «klassische» psychoanalytische Technik Freuds von ihren Vorläufern determiniert wird, aus welchen Elementen sie sich ihrerseits zusammensetzt. Es ist fraglich, ob wir die Technik der Psychoanalyse ohne eine solche Rekonstruktion ihrer Entstehung in ihrer systematischen Bedeutung überhaupt begreifen können. Wenn man den Ursprung der Freudschen Theorie der Technik abblendet und nur noch deren Ergebnis im Blick hat, gerät man in Gefahr, angesichts ihres großartigen Aufbaus den wichtigsten Stützpfeiler in ihrem Fundament zu vergessen: die Selbstanalyse.

\section{Anmerkungen}

${ }^{1}$ Daneben gibt es - vereinzelt - auch Überlegungen zur Selbstanalyse als relativ eigenständiger Methode der Introspektion: Farrow (1925, 1942) entwickelt in Anlehnung an die Psychoanalyse eine eigene Technik der Selbstanalyse, nach Horney (1942) kann die Selbstanalyse den psychoanalytischen Prozeß komplettieren und unter Umständen sogar ersetzen, Thomas (1972) propagiert die Selbstanalyse als Selbsthilfekonzept für Patienten, Ticho (1971) sieht die Selbstanalyse «als Ziel der psychoanalytischen Behandlung» an. Als einer der wenigen («orthodoxen») Psychoanalytiker hat Theodor Reik (1948) - ausgehend von der selbstanalytischen Traumdeutung Freuds - die eigene Selbstanalyse zum Ausgangspunkt einer Abhandlung über «die innere Erfahrung eines Psychoanalytikers» gemacht. Es wäre zu überlegen, inwiefern man die Selbstschilderungen von Tilman Moser $(1976,1979)$ als Resultate einer Selbstanalyse begreifen kann.

${ }^{2}$ Auf die Versuche, von neueren psychoanalytischen Positionen aus die einzelnen Krankengeschichten der «Studien über Hysterie» zu re-evaluieren, möchte ich hier nicht näher eingehen. Wahrscheinlich würde man heute einige der geschilderten Hysteriefälle als Borderlinezustände bzw. schizophrene Zustände charakterisieren. Siehe hierzu de Boor und Moersch (1980), die auf die einschlägigen Studien von Goshen (1952) und Reichard (1956) verweisen. Hirschmüller (1979, S. 133 f.) hält solche Versuche, «Paläodiagnostik» zu treiben, «weder für unproblematisch, noch für besonders fruchtbar».

${ }^{3}$ Um diese Axiome zu verdeutlichen, könnte man auch die Formeln aufstellen: Selbstanalyse = virtuelle Psychoanalyse; «Studien über Hysterie» = wichtigster vor-psychoanalytischer Text; psychoanalytische Technik = eindeutig festgelegte Behandlungsmethode der Psychoanalyse.

4 Nach Jones (1960, S. 373 ff.) beginnt Freud im Sommer 1897 mit der Selbstanalyse, die er bis zu seinem Lebensende fortsetzt.

${ }^{5}$ Diesem Ausdruck bin ich bei Theunissen (1965) begegnet, der ihn als philosophischen Begriff eingeführt hat. 
${ }^{6}$ In anderem Zusammenhang spricht Freud einmal von einer «Entfremdung zwischen dem Somatischen und dem Psychischen» (Freud, 1895, S.337), wobei er den Terminus «Entfremdung» eigens hervorhebt. Die Idee der Entfremdung bei Freud ist m. W. bisher noch nicht untersucht worden.

7 Diese «Druckprozedur» (Drücken mit der Hand auf die Stirn des Patienten) verweist direkt auf gewisse Techniken des Mesmerismus, bei dem durch bestimmten körperlichen Kontakt magnetische Kräfte vom Arzt auf den Patienten übertragen werden sollten. Freud bedient sich hier eines Kunstgriffes des tierischen Magnetismus, wahrscheinlich ohne sich darüber Rechenschaft abzulegen (siehe hierzu Zweig, 1931; Neyraut, 1974; Vliegen, 1976).

${ }^{8}$ Freud befaßt sich in einer späten Notiz eingehend mit den «Methoden» bzw. «Techniken» der Darstellung (Freud, 1940, S.141 f.).

${ }^{9}$ Diese Skizze wurde in einem Seminar in Zusammenarbeit mit Studenten entworfen.

\section{Literatur}

Abkürzung: GW für Freud, Sigmund: «Gesammelte Werke», Bd.I-XVIII. Frankfurt: S. Fischer, 1960-1968.

Anzieu, D. (1959): L'auto-analyse de Freud et la découverte de la psychoanalyse. Paris: Presse Universitaires de France, Nouvelle édition, 1975.

Bernfeld, S. (1946): An unknown autobiographical fragment by Freud. American Imago 4, S. 3-19.

Bernfeld, S., und S. Cassirer-Bernfeld (1944): Freud's early childhood. Bull. Menninger Clin. 8 (1944), S. 105-115.

de Boor, Clemens, und Emma Moersch (1980): Emmy von N. - eine Hysterie? Versuch einer Re-Evaluierung. Psyche 34, 265-279.

Brenner, Charles (1955): Grundzüge der Psychoanalyse. Frankfurt: S. Fischer, 1972.

Breuer, J., und S. Freud (1893): Über den psychischen Mechanismus hysterischer Phänomene. Vorläufige Mitteilung. In: Breuer, J., und S. Freud (1895a), Studien über Hysterie. GW I, 81-98.

Breuer, J., und S. Freud (1895): Studien über Hysterie. Frankfurt: Fischer Taschenbuch, 1970.

Breuer, J., und S. Freud (1895a): Studien über Hysterie. [Mit Auslassung der von Josef Breuer allein verfaßten Artikel: «Krankengeschichte Frl. Anna O. ...» und «Theoretisches»]. $G W$ I, 75-312.

Cremerius, Johannes (1979): Gibt es zwei psychoanalytische Techniken? Psyche 33, 577-599.

Farrow, E.Pickworth (1925): A method of self-analysis. The British Journal of Medical Psychology 5, 106-118.

- (1942): Psychoanalyse Yourself; a practical method of self-analysis enabling a person to remove unreasoning fears and depression from his mind. New York: International Universities Press, 1943.

Freeman, Lucy (1972): Die Geschichte der Anna O. Der Fall, der Sigmund Freud zur Psychoanalyse führte. München: Kindler Verlag, 1973.

Freud, S. (1895): Über die Berechtigung, von der Neurasthenie einen bestimmten Symptomenkomplex als «Angstneurose» abzutrennen. GW I, 313-342.

- (1900): Die Traumdeutung. GW II/III.

- (1908): Vorwort zur zweiten Auflage [der «Studien über Hysterie»]. GW I, $79 \mathrm{f}$.

- (1923): «Psychoanalyse» und «Libidotheorie». GW XIII, 209-233. 
Freud, S. (1933): Neue Folge der Vorlesungen zur Einführung in die Psychoanalyse. GW XV. - (1937): Die endliche und die unendliche Analyse. GW XVI, 57-99.

- (1940): Some elementary lessons in Psycho-Analysis. GW XVIII, 139-147.

Goshen, Charles E. (1952): The original case material of psychoanalysis. Amer. J. Psychiatr. $108,819-834$.

Greenson, R. R. (1967): Technik und Praxis der Psychoanalyse. Band I. Stuttgart: Klett, 1975.

Grunberger, Bela (1974): Von der «Aktiven Technik» zur «Sprachverwirrung». Studie zu Ferenczis Abweichung. In: Jahrbuch der Psychoanalyse. Beiträge zur Theorie und Praxis. Band XI, 100-124. Bern/Stuttgart/Wien: Hans Huber, 1979.

Hahn, Peter (1977): Das Konversionsmodell. In: Klinik der Gegenwart. Handbuch der Praktischen Medizin. München/Wien/Baltimore: Urban \& Schwarzenberg, Fassung 1977. Bd.IX, S. 540-548.

Hirschmüller, Albrecht (1979): Physiologie und Psychoanalyse in Leben und Werk Josef Breuers. Jahrbuch der Psychoanalyse/Beiheft 4. Bern: Hans Huber.

Horney, Karen (1942): Selbstanalyse. München: Kindler, 2. Aufl., 1976.

Jones, E. (1960): Das Leben und Werk Sigmund Freuds. Band 1. Bern/Stuttgart: Hans Huber.

Jung, C.G. (1921): Der therapeutische Wert des Abreagierens. «Gesammelte Werke» von C.G.Jung, 16. Bd., 137-147.

Kohut, H. (1974): Kreativität, Charisma, Gruppenpsychologie. Gedanken zu Freuds Selbstanalyse. Psyche 29, 1975, S.681-720.

Laplanche, J., und J.-B.Pontalis (1967): Das Vokabular der Psychoanalyse. Frankfurt: Suhrkamp, 1972.

Lipton, Samuel D. (1977): The advantages of Freud's technique as shown in his analysis of the Rat Man. Int. J. Psychoanal. 58, 255-273.

Mannoni, O. (1967): L'Analyse Originelle. Les Temps Modernes 22, 2136-2152.

Mitscherlich, Alexander (1975): Der Kampf um die Erinnerung. Psychoanalyse für fortgeschrittene Anfänger. München/Zürich: Piper.

Morgenthaler, Fritz (1978): Technik. Zur Dialektik der psychoanalytischen Praxis. Frankfurt: Syndikat.

Moser, Tilmann (1976): Gottesvergiftung. Frankfurt: Suhrkamp.

- (1979): Grammatik der Gefühle. Mutmaßungen über die ersten Lebensjahre. Frankfurt: Suhrkamp.

Neyraut, Michel (1974): Die Übertragung. Eine psychoanalytische Studie. Frankfurt: Suhrkamp 1976.

Pinel, Philippe (1809): Traité medico-philosophique sur l'aliénation mentale. 2. éd., entièrement refondue et très-augm. Paris: Brosson.

Reichard, Suzanne (1956): A re-examination of «Studies in Hysteria». Psychoanal. Quart. 25, 155-177.

Schur, M. (1973): Sigmund Freud. Leben und Sterben. Frankfurt: Suhrkamp.

Theunissen, Michael (1965): Der Andere. Studien zur Sozialontologie der Gegenwart. Berlin/New York: de Gruyter.

Thomä, Helmut (1979): In: Hildegund Fischle-Carl [Hrsg.] (1979): Theorie und Praxis der Psychoanalyse, 10-38. Fellbach: Bonz.

Thomas, Klaus (1972): Selbstanalyse. Die heilende Biographie, ihre Abfassung und ihre Auswirkung. Stuttgart: Georg Thieme. 
Ticho, Gertrude R. (1971): Selbstanalyse als Ziel der psychoanalytischen Behandlung. Psyche $25,31-43$.

Vliegen, Josef (1976): Von Mesmer bis Breuer. In: Die Psychologie des 20. Jahrhunderts. Bd.I [Herausgegeben von Heinrich Balmer]. S. 687-700. Zürich: Kindler.

Zweig, Stefan (1931): Die Heilung durch den Geist. Mesmer, Mary Baker-Eddy. Freud. Frankfurt: S. Fischer, 1966.

\section{Summary}

Sigmund Freud's first studies in psycho-pathology (especially his «Studies in Hysteria» published 1895 together with his teacher and friend Josef Breuer) were fundamental for his self-analysis. Every psycho-analysis is in itself a self-analysis, too.

Dr. med. Dr. phil. Heinz G. Schott

Institut für Geschichte der Medizin

der Albert-Ludwigs-Universität

Stefan-Meier-Straße 26

D-78 Freiburg i. Br. 